\title{
ASSESSING THE IMPACT OF DIGITALIZATION ON OPERATIONAL CAPABILITIES
}

\author{
Stefan Sommer, University of Duisburg-Essen, Germany
}

dx.doi.org/10.18374//JBS-21-1.1

\begin{abstract}
In traditional management thinking operational capabilities require to be based on valuable resources that have only limited substitutability and imitability. With the digitalization new company networks emerge and the individual network partners' operational capabilities are incorporated into the network and difficult to protect. How to capture the impact of digitalization on the requirements for these operational capabilities and how to explain the sustainable advantageousness of these capabilities is analyzed and empirically examined in a sample of 200 industrial companies. The analysis shows that more openness for value co-creation in networks is needed that can be economically sustainable within the network.
\end{abstract}

Keywords: Digitalization, operational capabilities, VRIN, environmental dynamics, transaction costs, ecosystems 